\title{
Über die autonome Kartierung der Umwelt durch lernende Serviceroboter
}

\author{
Herrn Prof. Dr. rer. nat. Dipl.-Ing. Henning Tolle zum 65. Geburtstag gewidmet
}

Andreas Kurz, Koblenz, und Georg von Wichert, Darmstadt

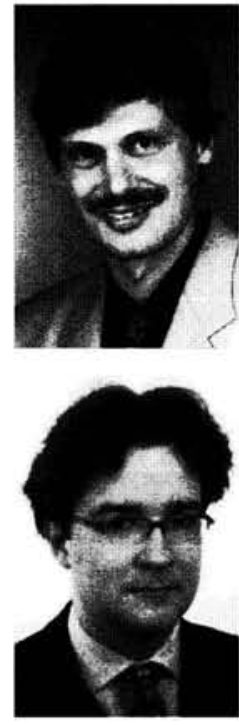

Prof. Dr.-Ing. Andreas Kurz lehrt an der Fachhochschule Koblenz Regelungstechnik, Automatisierungstechnik und Mathematik. In der angewandten Forschung licgen seine Schwerpunkte in den Bereichen Automatisierung, künstliche Intelligenz und Robotik.

Adresse: FH Koblenz, Fachbereich Elektrotechnik, Finkenherd 4, D-56075 Koblenz, Tel.: (0261) 9528136, Fax: (0261) 56953, E-Mail: kurz@fh-koblenz.de

Dipl.-Ing. Georg von Wichert ist wissenschaftlicher Mitarbeiter am Institut für Regelungstechnik der Technischen Hochschule Darmstadt, FG Regelsystemtheorie \& Robotik (Leiter: Prof. Dr. rer. nat. Dipl.-Ing. Henning Tolle). Er beschäftigt sich mit Fragen der Selbstorganisation bildbasierter Umweltrepräsentationen für autonome mobile Systeme.

Adresse: TH Darmstadt, Institut für Regelungstechnik, FG Regelsystemtheorie \& Robotik, Landgraf-GeorgStraße 4, D-64283 Darmstadt, Tel.: (06151) 16-4989, Fax: (06151) 16-2507, E-Mail: gvw@rt.e-technik.thdarmstadt.de

Mit der Verfügbarkeit immer leistungsfähigerer Sensoren, Aktoren und Rechner werden in naher Zukunft echte Serviceroboter eingesetzt werden. Sie werden in alltäglichen Bereichen agieren, die ein großes Maß an Selbständigkeit erfordern. Dazu bedarf es großer Flexibilität und Lernfähigkeit bei der Anpassung des Roboters an seine Einsatzumgebung. Nach einleitenden, prinzipiellen Überlegungen darüber, was eine intelligente, lernende Robotersteuerung leisten muß, wird in diesem Artikel besprochen, wie lernfähige Serviceroboter ihre Umwelt selbständig zu Navigationszwecken kartieren können. Entsprechende Forschungsergebnisse' zur Realisierung von dazu fähigen lernenden Robotersteuerungen mit Ultraschallsensoren und Kameras zur Wahrnehmung der Umwelt werden diskutiert.

\section{On autonomous map building by learning} service robots

Due to the increasing availability of powerful sensors, actuators and computers true service robots are currently becoming reality. They will be used for daily-life applications and will work in close cooperation with humans. This demands a high flexibility and learning capabilities for the robot to be able to adapt to

\footnotetext{
'Die Forschungsarbeiten wurden unterstützt durch die Deutsche Forschungsgemeinschaft (DFG, To-75/17-1/2, To-75/24-1) und das Bundesministerium für Bildung und Forschung (BMBF, ITR 8800 $\mathrm{B} / 5)$.
}

its environment. After some general considerations of a learning robot control problems we discuss how autonomous map building for learning service robots can be implemented. Finally some results concerning the realisation of such a learning control system for robots perceiving their environment with ultrasonic sensors or cameras are discussed.

\section{Einführung}

Miit der Verfügbarkeit immer leistungsfähigerer Sensoren, Aktoren und Rechner ist es mehr als nur eine utopische Vorstellung, daß Serviceroboter eingesetzt werden. Zuerst wohl, um den Menschen in gefährlichen Umgebungen zu ersetzen (z.B. bei der Minenräumung), später aber bestimmt auch, um Dienste im alltäglichen Lebensbereich zu leisten.

Diese Helfer werden sich in wesentlichen Punkten von den Handhabungsrobotern unterscheiden, die bereits in der industriellen Produktion eingesetzt werden. Vor allem werden sie in Bereichen agieren, die ein großes $\mathrm{Maß}$ an Selbständigkeit erfordern. In den meisten Fällen werden sie mobil sein, also einen relativ großen Aktionsbereich haben und oft in unmittelbarer Nachbarschaft mit dem Menschen eingesetzt werden können, ohne daß dabei besondere Sicherungsvorkehrungen getroffen werden müssen. Besonders wichtig ist es auch, daß sie in der Lage sein müssen, mit technischen Laien zusammenzuarbeiten. Es muß also genügen, ihnen die gewünschte Aufgabe nur grob zu schildern, so wie man es gegenüber einem menschlichen Bediensteten auch tun würde. Es ist klar, daß dies eine gewisse Intelligenz voraussetzt (im ursprünglichen Wortsinn: die Fähigkeit, fehlende Elemente ,dazwischenlegen“ zu können). Es ist leicht einzusehen, daß dafür die Maschinen eigene Zugänge zur Welt über leistungsfähige Sensoren und eine entsprechend gute Informationsverarbeitung haben müssen.

Wie die beiden wesentlichen Fähigkeiten - Mobilität und Kommunikation auf hohem Abstraktionsniveau realisiert werden können, kann bereits anhand der einfach erscheinenden Aufgabe, nämlich den Befehl „Fahre von Ort A nach Ort B!“" auszuführen, in allen wesentlichen Aspekten untersucht werden. Da in irgendeiner Form jeder mobile Serviceroboter diese Aufgabe lösen können muß, ist sie eine Schlüssel- oder Basisaufgabe. 
Bei der Fabrikautomatisierung werden bereits jetzt fahrerlose Transportsysteme (FTS) eingesetzt, die diese Basisaufgabe zuverlässig lösen können. Allerdings wird ihre Umwelt speziell hergerichtet, z.B. werden Induktionsdrähte im Boden verlegt. Zukünftige Serviceroboter müssen sich jedoch auch in Umgebungen zurechtfinden können, die im vorhinein nicht genau bekannt sind. Der Nutzer möchte auch möglichst keine teuren oder umständlichen Vorkehrungen treffen müssen, um sicherzustellen, $\mathrm{da} \beta$ der Roboter sich zurechtfindet. Vielmehr ist es wünschenswert, daß die vorhandenen Strukturen der Umwelt zur Orientierung ausreichen. Dann treten im Vergleich zum induktionsdrahtgeführten FTS wesentlich größere Probleme auf, die bis heute noch nicht befriedigend gelöst sind. Es existieren zwar bereits sehr vielversprechende Ansätze, insbesondere zur bildbasierten Navigation (z.B. [1], [2] oder [3]), die aber meist ein aufwendig zu erstellendes Modell der Umgebung benötigen oder aber nur einfache Elementaraktionen durchführen können [4]. Darüber hinaus existiert noch ein Vielzahl anderer Systeme, die mit Ultraschall-, Laserdistanz- oder Radarsensoren ihre Umwelt erfassen. Hier werden zumeist entweder Gitterrepräsentationen der Umwelt ermittelt ([5] und viele daraus abgeleitete Methoden) oder geometrische Modelle der Umwelt und in ihr enthaltener Objekte aufgebaut [2].

In jedem Fall müssen leistungsfähige Sensoren verwendet werden. Diese liefern große Datenmengen, weshalb eine entsprechend aufwendige Datenverarbeitung nötig ist, die aus der Datenflut genau die Information herausfiltern kann, welche für die Lösung der Aufgabe benötigt wird.

Im folgenden werden zunächst prinzipielle Überlegungen darüber angestellt, was eine intelligente und vor allem lernende Robotersteuerung zur Lösung der genannten Basisaufgabe leisten muß (Kapitel 2). Es wird insbesondere gezeigt, da $\beta$ das Erlernen einer Umweltkarte eine wesentliche Fähigkeit intelligenter mobiler Roboter sein muß. Im nächsten Kapitel wird ein Ansatz für die lemende Umweltkartierung vorgestellt und diskutiert, wie weit er führt, wenn ein Ring von Ultraschalldistanzsensoren benutzt wird, welcher laufend radarähnliche Bilder der Umgebung liefert. Dabei werden Argumente dafür gewonnen, da $B$ das Pendant zum menschlichen Auge - eine Kamera - nötig ist, um Serviceroboter ausreichend leistungsfähig zu machen (Kapitel 3). Im letzten Kapitel wird gezeigt, daß basierend auf denselben Prinzipien auch eine bildverarbeitende Lösung konstruiert werden kann. Entsprechende Forschungsergebnisse werden im Vergleich zu der Lösung mit Ultraschallsensoren diskutiert.

\section{Lernebenen}

Um die genannte Basisaufgabe lösen zu können, also

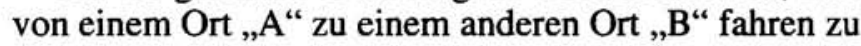
können, muß der mobile Roboter den Namen „A“ und „B“ innere Bilder (Ortsrepräsentationen) zuordnen können, welche diesen Orten entsprechen. Außerdem muß er Wissen gespeichert haben, aus dem er ableiten kann, wie er von dem einen Ort zum anderen kommen kann, welche konkreten Aktionen er dazu durchführen muß (Topologiewissen).

- Ein FTS muß beispielsweise Wissen über Verzweigungen und bestimmte Stationen (Bearbeitung, Parken usw.) gespeichert haben (Ortspräsentationen). Es muß außerdem Information darüber besitzen, welche Orte durch Leiteinrichtungen verbunden sind und welche Richtungen an einer Wegkreuzung oder an einer Weiche eingeschlagen werden können (Topologiewissen).

- Ein Mensch besitzt Gedächtnisinhalte, um Orte mit Hilfe seiner Sinnesorgane (überwiegend mit den Augen) wiedererkennen zu können (Ortsrepräsentationen). Er weiß auch darüber Bescheid, zwischen welchen Orten ein Übergang möglich ist und wie man eine Distanz überwinden kann, z.B. von München nach Berlin mit dem Zug/Auto unter Zuhilfenahme weiterer Informationsquellen (Autobahnkarten, Fahrpläne) oder von einem Raum in den anderen; wo ist eine Tür? (Topologiewissen).

Ortsrepräsentationen und Topologiewissen bilden zusammen eine Umweltkarte. Diese muß erlernt werden, wenn der Serviceroboter auch in Umwelten agieren können soll, die ihm zunächst unbekannt sind. Speziell für das Erlernen von Ortsrepräsentationen ist es sinnvoll, dem mobilen Roboter eine Sensordatenvorverarbeitung vorzugeben, die grob auf seine sensorischen Möglichkeiten und den zu erwartenden Umwelttyp angepaßt ist. Gerade bei den erforderlichen leistungsfähigen Sensoren wird jedoch das Wechselspiel zwischen ihnen und der Umwelt und die sensorisch wahrnehmbaren Eigenschaften der Umgebung im Vorfeld des Einsatzes schwer zu übersehen sein. Deshalb muß der Roboter diese Anpassung der Sensordatenverarbeitung möglichst selbst vornehmen können. Aus dieser Sicht ist das Lernen auf der niederen Ebene der Sensordatenvorverarbeitung und Merkmalsextraktion von großer Bedeutung.

Ein gegebener Fahrbefehl muß natürlich, bevor er ausgeführt werden kann, verstanden werden. Dazu ist ein weiterer Lernvorgang notwendig, bei dem die inneren Ortsrepräsentationen mit den Symbolen der Befehlssprache verbunden werden (was bedeuten „Raum A“, „Raum B“, was ist überhaupt ein „Raum“?). Auch alle anderen Symbole müssen entsprechendem Wissen zugeordnet werden (was bedeutet „Fahren“, „Andocken“ usw.).

Ist der Befehl verstanden worden, soll er ausgeführt werden. Dazu müssen geeignete Verhaltensweisen und Prozeduren verfügbar sein, die in der Karte enthaltene Informationen benutzen können. Intelligente mobile Systeme werden in der Lage sein müssen, auch auf dieser Ebene zu lernen.

- Ein FTS muß zur Lösung der Basisaufgabe beispielsweise einer Fahrspur bis zur nächsten Weiche folgen. Der Mensch wird für sein Verhalten in einer entsprechenden Situation gelernt haben, die richtige Tür im Raum zu suchen, sich dieser zu nähern und dann hindurchzugehen. 
Meist werden dabei Regelkreise geschlossen. Die Information, die in der Umweltkarte gespeichert werden muß, hängt von der Art der Aufgabenstellung ab.

- Beispielsweise wird ein Roboter, der in einem Krankenhaus Medizin oder Mahlzeiten verteilen soll, die einzelnen Zimmer und Krankenbetten auseinanderhalten müssen. Ein Inspektionsroboter für Abwassertunnel muß das Tunnelnetz kennen...

Zusammenfassend können folgende Ebenen des Lernens unterschieden werden:

1. Erlernen von internen Ortsrepräsentationen mit Hilfe einer vorgegebenen Sensordatenvorverarbeitung, die grob auf den zu erwartenden Umwelttyp angepaßt ist. (Die vorgegebene Sensordatenverarbeitung entspricht in der biologischen Evolutionstheorie dem, was die Art, der Genotyp, erlernt: eine Methode, wie der Phänotyp am besten lernt).

2. Erlernen einer Umweltkarte, wofür interne Ortsrepräsentationen mit Topologiewissen verbunden werden.

3. Erlernen der Befehlssprache, die zur Kommunikation mit dem Auftraggeber (z.B. einem technischen Laien) benötigt wird.

4. Erlernen von Fähigkeiten, Regeln und Heuristiken, die benötigt werden, um die Aufgabe durchführen zu können.

Diese Ebenen ergeben sich nicht nur bei der Basisaufgabe für mobile Systeme, sondern auch bei beliebigen anderen Aufgaben, die ein Agieren im Raum erfordern.

\section{Lernende Steuerung eines mobilen Roboters auf der Basis von Ultraschallmessungen}

Im diesem Kapitel wird für einen konkreten mobilen Roboter (Bild 1) ein Konzept zur lemenden Umweltkartierung vorgestellt. Dieses wurde realisiert und experimentell untersucht (eine genauere Darstellung findet man in der Dissertationsschrift [6]). Vor dem Hintergrund der dabei gewonnenen Erfahrungen werden die beiden ersten der aufgelisteten Lernebenen diskutiert. Dabei wird herausgearbeitet, warum es von Vorteil ist, bildverarbeitende Steuerungen für mobile Roboter vorzusehen.

Der verwendete Experimentier-Roboter besa $\beta$ als Hauptsensorik einen Ring von 24 distanzmessenden Ultraschallsensoren, mit dem er zwei radarähnliche Bilder pro Sekunde aufnehmen konnte. Außerdem hat er an den beiden Motorachsen (Rotation und Translation) zwei Goniometer, deren Meßsignale für die Navigation herangezogen werden konnten.

\subsection{Erlernen von Ortsrepräsentationen (Situationsgebiete)}

Es ist klar, daß die von dem Ultraschallsensor-Ring gelieferte Datenmenge zu groß ist, um direkt weiter-

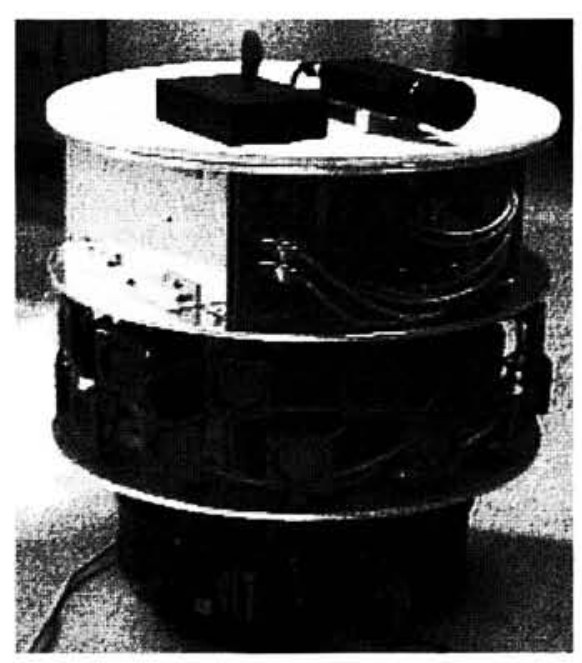

Bild 1: Der mob Rotober ALEF.

verarbeitet werden zu können. Die Daten müssen so verdichtet werden, daß sie Orten im Bewegungsraum des Roboters zugeordnet werden können. Die meisten Ansätze sehen vor, bestimmte Strukturen der Umwelt zu erkennen (z.B. Kanten von Gegenständen, Wände, Türöffnungen usw.). Dies entspricht der menschlichen Objekterkennung aus Bildern, die sicherlich eine Realität ist.

Parallel dazu wird vom Menschen jedoch eine weitere Art der Bildauswertung genutzt: Bestimmt hat jeder einmal einen Ort klar wiedererkannt und dabei gleichzeitig wahrgenommen, daß sich irgendetwas verändert hat, ohne dieses genau benennen zu können. In diesen Fällen findet Erkennen offensichtlich aufgrund eines Gesamteindrucks statt. Beispielsweise ist das Raumgefühl, also die Einordnung des eigenen Standorts im Raum, dieser zweiten Wahmehmungsart zuzuordnen.

Bei der Einschätzung, ob man z.B. mitten im Raum, in einer Ecke oder in der Nähe einer Wand steht, muß vieles gleichzeitig in Betracht gezogen werden. Die Objekterkennung spielt hier eine untergeordnete Rolle.

Die im folgenden vorgestellte Methode zur Auswertung der Ultraschalldaten entspricht dieser ganzheitlichen Betrachtungsweise. Sie sieht deshalb in der Konsequenz vor, die Ultraschall-Radarbilder als Ganzes zu klassifizieren und so bestimmten Situationen zuzuordnen.

Das Bild 2 zeigt die verschiedenen Stufen der Datenverdichtung. Vor der Klassifikation werden zu große Distanzmeßwerte auf einen maximalen Wert begrenzt, um so die lokale Situation besser erfassen zu können, eine Lipschitz-Filterung verringert Störeinflüsse und es wird - als wichtigste Vorverarbeitungsoperation - eine virtuelle Verdrehung des Sensorringes durchgeführt. Dabei werden die Meßwerte zyklisch umnumeriert und so dasselbe erreicht, wie wenn der Sensorring in eine bevorzugte Richtung gedreht würde. Auf diese Weise ist es möglich, die Erkennung einer Situation von der Orientierung des Roboters im Raum unabhängig zu machen.

Bei der Klassifikation werden lernende Verfahren eingesetzt (erprobt wurden RCE-Klassifikator [7] und 


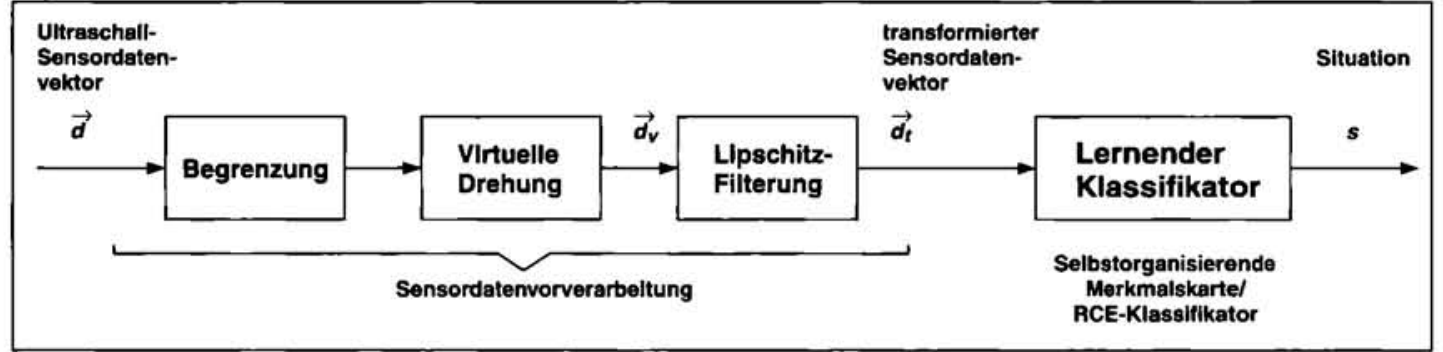

Bild 2: Klassifikationssystem zur Erkennung von Situationen.

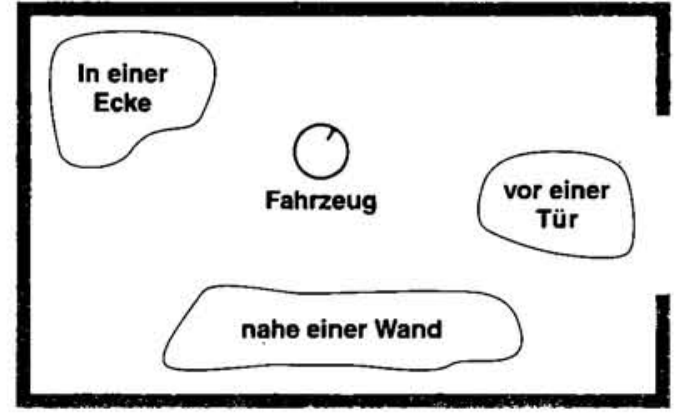

Bild 3: Veranschaulichung der Idee der Situationsgebiete.

Kohonennetz [8]). Dadurch wird die Intelligenz und Flexibilität des Roboters entscheidend erhöht: Er kann auf diese Weise Anzahl und Art der unterschiedenen Situationen selbständig auf seine Sensorik und die Umwelt anpassen.

$\mathrm{Da}$ die Ultraschallbilder in einem bestimmten lokal begrenzten Bereich ähnlich sein werden, ist zu erwarten, $\mathrm{da} B$ der Klassifikator dort immer dieselbe Situation erkennt, da $B$ also Situationsgebiete entstehen, ähnlich solchen, wie sie auch ein menschlicher Beobachter definieren würde (siehe Bild 3). Genauer wird der gesamte Bewegungsraum des Roboters in solche Situationsgebiete zerlegt, wie das in Experimenten gewonnene Bild 4 (links) zeigt. Damit ist das Ziel erreicht, bestimmten Orten interne Repräsentationen (hier: die Daten für die interne Darstellung von Situationsgebieten) zuordnen zu können.

\subsection{Aufbau einer Umweltkarte (Situationskarte)}

Die Umweltkarte verbindet Ortsrepräsentationen mit Topologiewissen. Dies bedeutet hier, daß etwas darüber gelernt werden muß, wie man von einem Situationsgebiet in das nächste kommen kann. Am besten gelingt dies, indem ein Übergangsgraph aufgebaut wird, im folgenden (Situationskarte) genannt: Die Situationsgebiete entsprechen den Knoten und mögliche Übergänge den Kanten des Graphen. Der Lernvorgang findet statt, während der Roboter durch seine Umgebung fährt, getrieben von einer implementierten Neugierkomponente seiner Steuerung. Jedesmal, wenn er neue Situationsgebiete oder UUbergänge findet, erweitert er die Situationskarte um entsprechende Knoten und Kanten.

Dieser Vorgang wäre unproblematisch, wenn die Situationsgebiete durch die Klassifikation eindeutig erkennbar wären. Dies ist jedoch nicht der Fall: es gibt viele Situationsgebiete, die bei der Klassifikation der Ultraschalldaten nicht unterschieden werden können, in denen also die gleiche Situation erkannt wird. Um diese auseinanderhalten zu können, müssen weitere Merkmale herangezogen werden. Ein solches ist die Lage eines Situationsgebiets in einem Umweltkoordinatensystem. Sie kann prinzipiell aus den Goniometriedaten der Winkelgeber an den Motorachsen durch Koppelnavigation bestimmt werden. Die Koppelnavigation ist jedoch mit Driftfehlem behaftet: Die Positionsbestimmung wird ohne laufende Korrektur schnell so ungenau, da $\beta$ sie nicht mehr zu gebrauchen ist. Wenn eine Umweltkarte vorhanden ist, können die Fehler der Koppelnavigation durch Vergleich mit den eingezeichneten Ortspositionen zwar korrigiert werden. Es stellt sich aber dann das Grundproblem der Kartierung:

Zum Erlernen einer Umweltkarte werden Ortspositionen (hier: Lagen von Situationsgebieten) benötigt, die durch Koppelnavigation berechnet werden. Die Fehler der Koppelnavigation wiederum werden aufgrund der in der zu lernenden Karte verzeichneten Ortspositionen eliminiert.

Positionsbestimmung und Navigationsfehlerkorrektur stehen also in einer wechselseitigen Abhängigkeit. Die Lösung dieser Problematik ist wesentlich und nicht trivial. Für den Experimentierroboter konnte es für nicht zu große Umwelten gelöst werden, indem Kalmanfiltertechniken eingesetzt wurden. Das Bild 4 (rechts) zeigt eine Situationskarte, die auf diese Weise aufgebaut wurde. Es konnte auch eine Situationskarte einer Um- 
welt mit einer Fläche von ca. $200 \mathrm{~m}^{2}$ Fläche erfolgreich gelernt werden (Bild 5). Bei dieser Größe dürfte jedoch das Verfahren nach den gewonnenen Erfahrungen nicht mehr weit von der Grenze entfernt sein, an der der Kartierungsproze $B$ instabil wird.

Generell muß zur Lösung des Kartierungsproblems angestrebt werden, möglichst viele verschiedene Strukturen zu unterscheiden, die aber gleichzeitig möglichst gut wiedererkannt werden können.

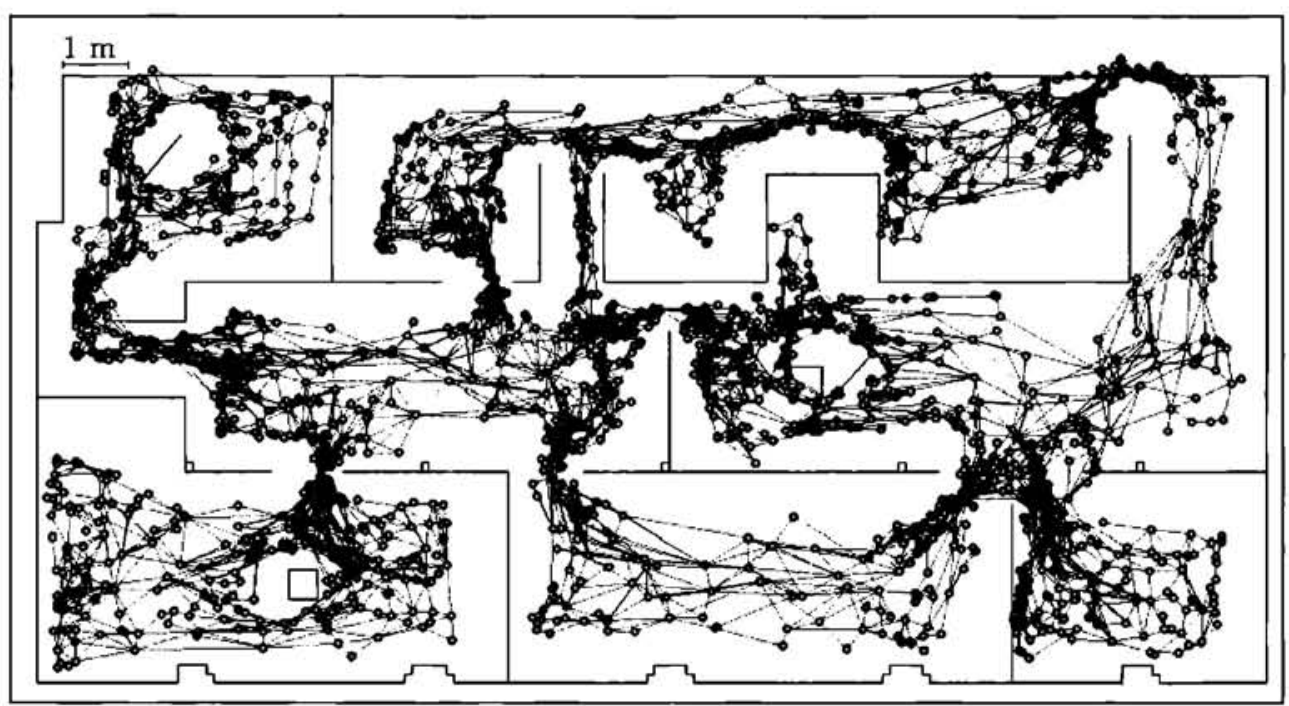

Bild 5: Situationskarte einer Umwelt von etwa $200 \mathrm{~m}^{2}$ Fläche.
Diese beiden Ziele stehen

in Konkurrenz zueinander. Es ist leicht, die lernende Klassifikation so einzurichten, daß mehr Situationen unterschieden werden. Aber im Gegenzug werden die Situationsgebiete immer kleiner, der Anteil der Übergangsgebiete von einem Situationsgebiet zum anderen, in denen keine eindeutige Klassifikation möglich ist, wird schnell größer, die Wiedererkennbarkeit sinkt.

Man erkennt das Prinzip, wenn man sich klarmacht, daß beides, Wiedererkennbarkeit und Erkennungsvielfalt, Anforderungen an den Umfang der Information stellen, die die Sensorik liefern muß: deshalb kann nur ein Kompromiß gefunden werden. Grundsätzlich läßt sich demzufolge die Grenze, innerhalb derer das Kartierungsproblem gelöst werden kann, nur erweitem, wenn die Bandbreite der Sensorik erhöht wird.

In der Natur haben die meisten intelligenten Lebewesen Augen. Auch der Mensch kompensiert seine mangelnde Fähigkeit, die Umwelt zu vermessen, dadurch, daß er aufgrund des visuellen Eindrucks nahezu unbegrenzt viele Orte eindeutig wiedererkennen kann. Der Einsatz einer Kamera verspricht also eine weitgehende Verbesserung bei Lösung des Kartierungsproblems.

\section{Lernende Bildverarbeitung für Navigationszwecke}

Im vorhergehenden Abschnitt wurde gezeigt, daß Basisaufgaben, wie die Navigation in unbekannter Umgebung nicht notwendigerweise einer objektbezogenen Interpretation der Sensordaten bedürfen, vielmehr wurden die jeweiligen Meßwerte der Ultraschallsensoren als Merkmale der Situation verstanden, in welcher sich das System zum gegenwärtigen Zeitpunkt befand. In der Umweltkarte wurden die Situationsmerkmale der jeweiligen Raumbereiche vermerkt. Es wurde diskutiert, daß die ultraschallbasiert ermittelten Merkmale in großen Umgebungen nicht reichhaltig genug sind, um die auftretenden Mehrdeutigkeiten aufzulösen.
Deshalb soll in diesem Abschnitt gezeigt werden, wie sich analog Situationsmerkmale aus Bilddaten, die ein größeres Potential zur Situationsdifferenzierung haben, ermitteln lassen. Anders als bei den herkömmlichen modellbasierten Ansätzen, die das Vorhandensein modellierter Umgebungsstrukturen (Objekte, Wände etc.) voraussetzen, wird hier ebenfalls ein unüberwacht lernender Ansatz verfolgt. Dabei wird - wie oben - auf eine Objekterkennung anhand der Sensordaten verzichtet. Dies hat den Vorteil, daß einerseits der sonst erforderliche Modellierungsaufwand vermieden wird, andererseits paßt das System seine Wahrnehmung in einem Lernprozeß selbst an die - a priori meist unbekannten - sensorisch erfaßbaren Charakteristika seiner Umwelt an.

\subsection{Unüberwachte Extraktion von \\ Situationsmerkmalen aus Bildern}

Wie in Bild 6 dargestellt, besteht der Prozeß zur unüberwachten Extraktion von Situationsmerkmalen aus den Kamerabildern des Roboters im wesentlichen aus drei Verarbeitungsstufen:

1. Im ersten Schritt werden lokale, pixelbezogene Merkmale berechnet, und so ein mehrkanaliges Merkmalsbild erzeugt. Im Fall von Graustufenbildern werden dies Merkmale sein, die den jeweiligen Bildpunkt anhand der Grauwertänderungen in seiner Umgebung charakterisieren. Besitzt das System eine Farbkamera, so können hier statt dessen (oder auch zusätzlich) aus den Farbwerten abgeleitete Merkmale verwendet werden. In beiden Fällen ist darauf $\mathrm{zu}$ achten, daß eine möglichst starke Unabhängigkeit der Merkmale von der absoluten Bildhelligkeit besteht, um Beleuchtungseffekte zu unterdrücken.

2. Der zweite Schritt der Verarbeitung besteht in einer lernenden Quantisierung der Pixelmerkmale, aus der eine unüberwachte Segmentierung des Bildes resultiert. Diese faßt Gebiete ähnlicher lokaler Merkmale zusammen. Hier sind verschiedenste Methoden denkbar, jedoch hat sich eine hierarchische Anordnung zweidimensionaler Kohonennetze bewährt [9]. 


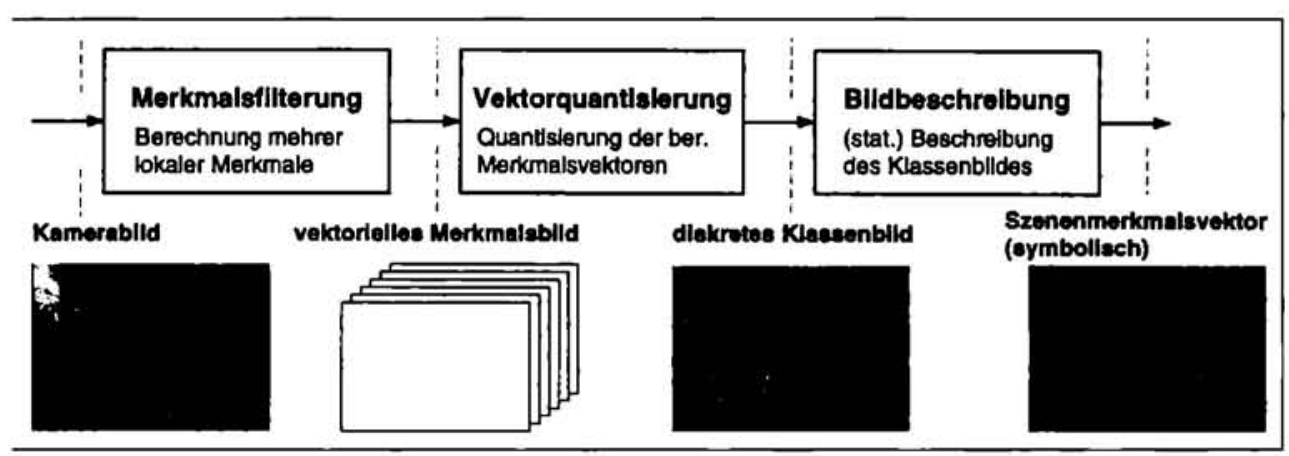

Bild 6: Grobstruktur des Prozesses zur unüberwachten Extraktion von Situationsmerkmalen aus Videobildern.
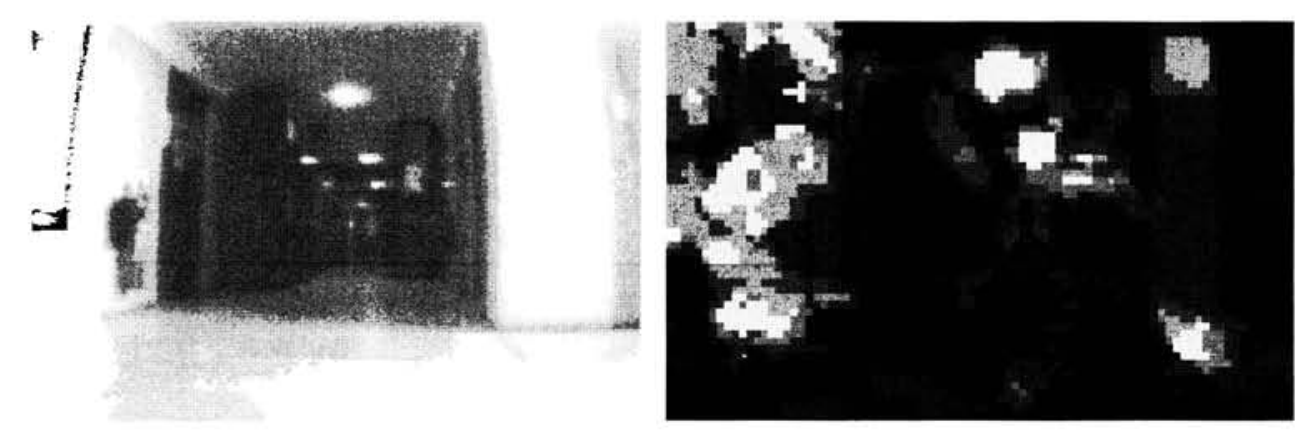

Bild 7: Bild der Onboardkamea und zugehöriges Segmentierungsergebnis.

Im Ergebnis erzeugen die beiden ersten Stufen der Verarbeitung ein unüberwacht segmentiertes Bild (siehe Bild 7). Es ist aufgrund der lokalen Eigenschaften der zur Segmentierung verwendeten Merkmale robust gegenüber leichten Verschiebungen des Kamerastandortes (und damit der Roboterposition). Diese Robustheit ermöglicht es, im nächsten Schritt aus diesem Klassenbild Szenenmerkmale zu ermitteln, welche sich während der Roboterbewegung weitestgehend kontinuierlich ändern. Dies ist erforderlich, um aus ähnlichen Szenenmerkmalen auf ähnliche Situationen schließen zu können.

3. Schritt drei besteht aus der Bestimmung von Merkmalen, welche die Verteilung der unterschiedlichen Gebiete im Klassenbild beschreiben. Diese Verteilung ist vom Bildinhalt und damit von der gegenwärtigen Situation des Roboters abhängig. Dabei erfaßt ein Bild selbstverständlich nur einen Ausschnitt der Gesamtsituation. Hier lassen sich ebenfalls beliebige viele Merkmale denken (siehe dazu z.B. [10]). Gute Resultate werden jedoch vor allem mit den aus der Formerkennung bekannten geometrischen Momenten [11] erzielt. Ihr Vorteil gegenüber anderen Formmerkmalen, wie z.B. Fourierdeskriptoren, besteht darin, da $\beta$ die Klassengebiete zur Berechnung der Momente nicht zusammenhängen müssen, dies wäre eine Forderung, die die unüberwachte Segmentierung durch Quantisierung lokaler Bildpunktmerkmale nicht erfüllen kann.

Die berechneten Szenenmerkmale können, zu Merkmalsvektoren zusammengefaßt, die Basis für einen Vergleich verschiedener Bilder ohne Interpretation des Bildinhaltes bilden.
4.2 Ergebnisse zur Beurteilung der unüberwacht extrahierten Merkmale

In diesem Abschnitt sollen die Eigenschaften der berechneten Szenenmerkmale und ihre Eignung in dem hier betrachteten Zusammenhang diskutiert werden.

Wie schon angedeutet, kann der Abstand der Merkmalsvektoren zweier Bilder als Ähnlichkeitsmaß für den optischen Gesamteindruck der Bilder interpretiert werden. Mit Hilfe einer lernenden Klassifikation oder Vektorquantisierung, die Gruppen von Bildern in Abhängigkeit ihrer Merkmale zusammenfaßt und dabei die Verteilung der Merkmalsvektoren im Merkmalsraum berücksichtigt, soll gezeigt werden, $\mathrm{da} B$ die vom System in einem unüberwachten Vorgang ermittelten Merkmale tatsächlich den optischen Gesamteindruck der Bilder charakterisieren und damit geeignet sind, die Ultraschalldatenvektoren aus Abschnitt 3 zu ersetzen.

Für die lernende Klassifikation der Szenenmerkmalsvektoren wurden Growing Neural Gas-Netzwerke [12] verwendet. Anders als bei Kohonennetzen muß hier die inhärente Dimensionalität der Daten ${ }^{2}$ nicht bekannt sein, um topologische Defekte des trainierten Netzes zu vermeiden.

Die zugrundeliegenden Experimente wurden in einer normalen Büroumgebung durchgeführt. Diese wurde in keiner Weise für den Roboter präpariert. In Bild 8 wird die Fahrt des Roboters durch die Umgebung dargestellt. Die Pfeile repräsentieren Position und Orientierung des Roboters im Moment der Bildaufnahme, die Farbe (Grauwert) des jeweiligen Pfeils kodiert die Klassenzugehörigkeit des jeweiligen Bildes. Man erkennt eine Partitionierung der Umgebung wie in Abschnitt 3, wobei hier natürlich eine Abhängigkeit von der Orientierung besteht. Dennoch gibt es größere Bereiche, in denen die Bilder auch bei verschiedenen Durchfahrten desselben Gebietes einer bestimmten Klasse zugeordnet werden.

Betrachtet man sich nun die Bilder, so zeigt Bild 9 deutlich, daß die Ähnlichkeit der Bilder innerhalb der jeweiligen Klasse größer ist, als zwischen Bildern verschiedener Klassen. Die den Klassen zugeordneten Bedeutungen „Engstelle“ und „Freiraum“ sind natürlich willkürlich und werden vom System nicht berück-

${ }^{2}$ Hier ist die Anzahl der Freiheitsgrade der Verteilung der Merkmale im Gegensatz zur Dimension der Merkmalsvektoren, die natürlich feststeht und bekannt ist, gemeint. 
sichtigt. Hier wurde die Anzahl der Klassen (Neuronen im Netz) auf 3 begrenzt. Die dritte Klasse enthält Bilder, welche eine kompliziertere Struktur haben, als die der 2 hier gezeigten Klassen.

Erlaubt man dem System eine feinere Differenzierung der Bilder (16 Klassen) so spezialisieren sich einzelne Klassen auf bestimmte Bildtypen (beispielsweise das Regal in Bild 10), die selbst aus sehr unterschiedlicher Perspektive erkannt werden. Dieser Effekt läßt sich auch in Bild 11 zeigen.

Der Roboter fährt hier in einem Korridor. Wird die Anzahl der Klassen auf 15 beschränkt, so werden Ansichten in beiden Richtungen des Korridors zusammengefaßt und derselben Klasse zugeordnet. Werden jedoch 50 Klassen zugelassen, so werden die Fahrtrichtungen unterschieden, ohne $\mathrm{da}$ die Bildgruppen in kleinere Einheiten zerfallen. Dies kann als Hinweis darauf verstanden werden, daß die berechneten Szenenmerkmale die Eigenschaften von Umgebung und Sensorik in angemessener Weise berücksichtigen und die Nachbarschaftsverhältnisse der Bilder bezüglich ihrer Ähnlichkeit im Gesamteindruck im Merkmalsraum widergespiegelt werden.

Aufgrund der unüberwachten Klassifikation ist eine Interpretation der in Bild 8 gezeigten Partitionierung der Umwelt nicht zulässig. Die Bilder 9 und 10 dokumentieren jedoch, $\mathrm{da} \beta$ die Ergebnisse auch dem Menschen plausibel scheinen.

Die benötigte Rechenzeit für das Verfahren beträgt auf einem SPARC-Prozessor (SPARC 20, 60 MHz) etwa $1,6 \mathrm{~s}$. Durch einfache Optimierungen kann dies aber noch weiter reduziert werden.

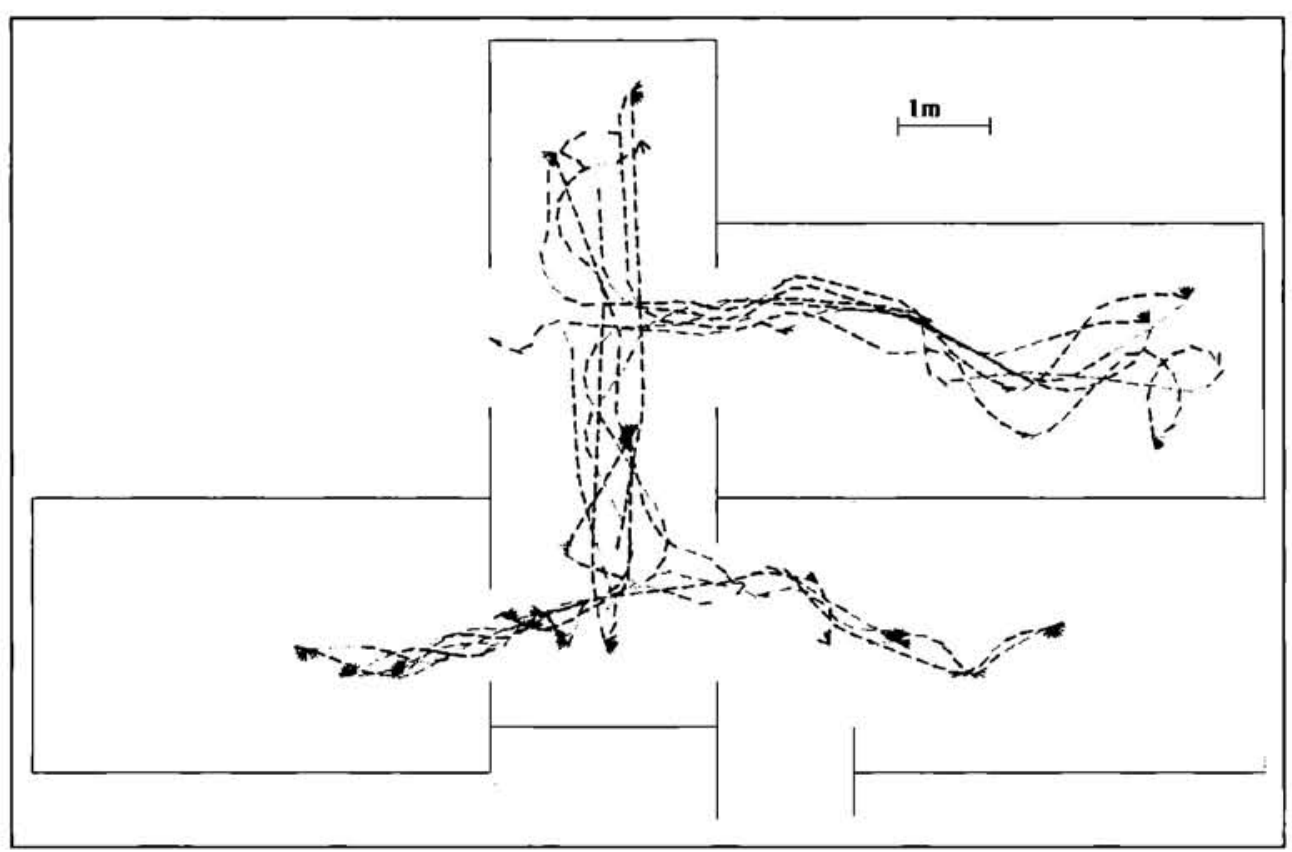

Bild 8: Fahrt des Roboters durch die Büroumgebung.
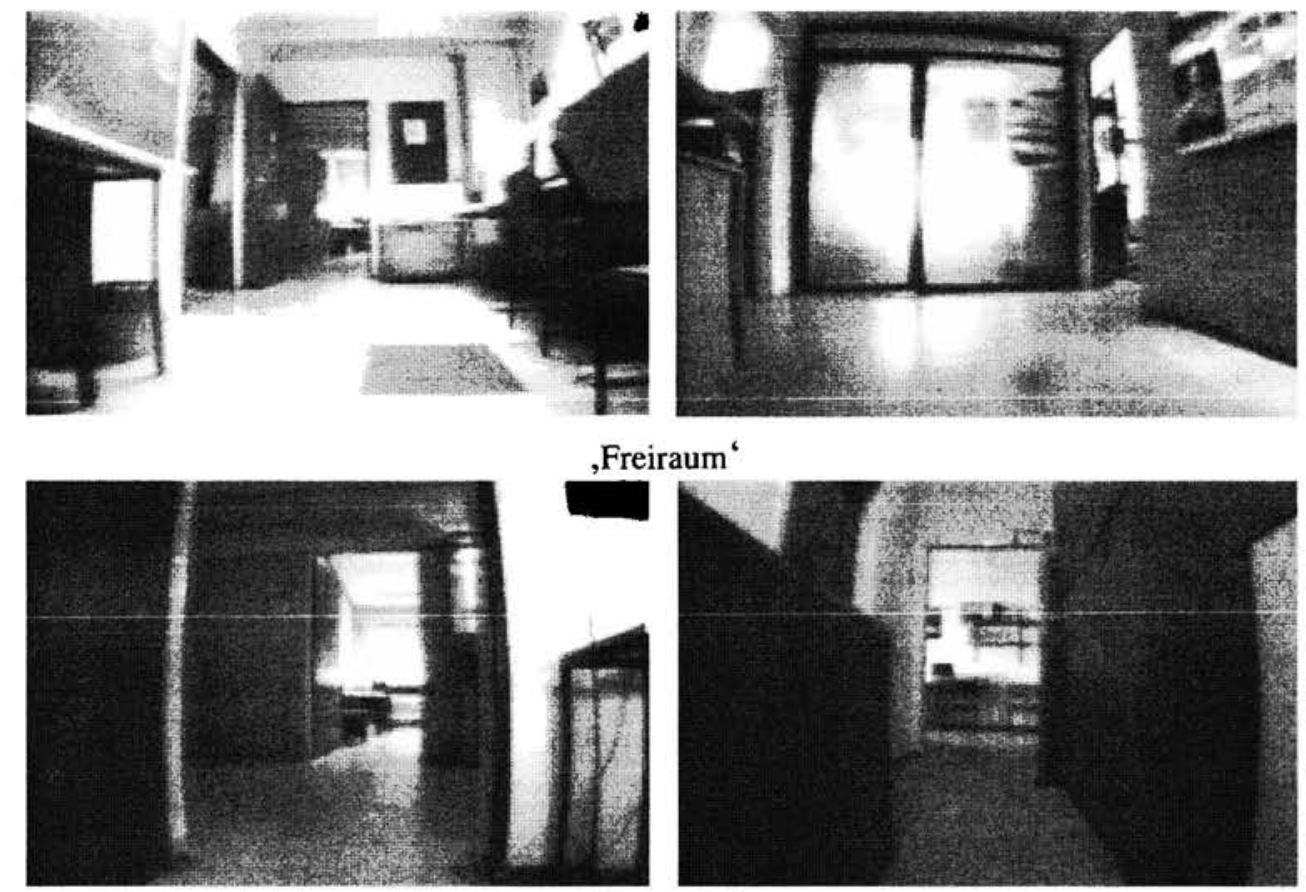

,Engstelle ‘

Bild 9: Grobe Unterscheidung von „Engstelle“ und „Freiraum“ mit wenigen Klassen.
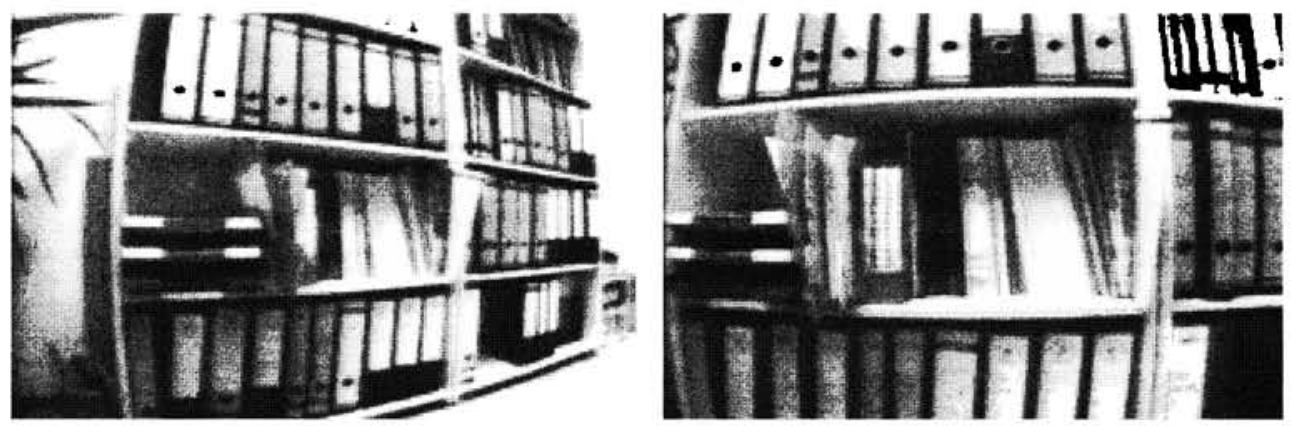

Bild 10: Bilder einer spezialisierten Klasse. 


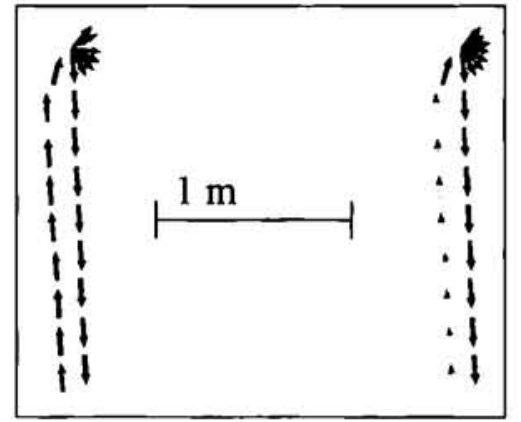

Bild 11: Ausschnittvergrößerung der Fahrt durch die Büros (Flurszene). Bei einer groben Klassifikation werden Bilder beider Fahrtrichtungen nicht unterschieden (links). Eine feinere Klassifikation kann sie jedoch trennen.

\subsection{Vergleich von bild- und ultraschallbasierter Ortsrepräsentation}

In den vorhergehenden Abschnitten wurde gezeigt, daß sowohl aus Ultraschalldaten als auch auf der Basis von Videobildern selbstorganisierend interne Ortsrepräsentationen aufgebaut werden können.

Vergleicht man die beiden, so enthalten, wie erwartet, die Bilder gerade im Alltagsumgebungen mehr Information, als die reinen Distanzdaten. Sie besitzen daher ein größeres Potential zur Situationsdifferenzierung. Es wurde oben bereits diskutiert, daß dies bei großen Karten erforderlich ist, soll der Kartenaufbauprozeß stabil bleiben. Darüber hinaus sind die Bilder sehr viel orientierungsselektiver, als die Messungen des Ultraschallsensorringes. Dies ist deshalb von besonderem Wert, weil die Koppelnavigationsfehler in der Hauptsache bei Drehungen des Roboters entstehen.

Allerdings sind die „Messungen“ einer Kamera noch mehr als die Ultraschallmessungen von den Eigenschaften der konkreten Einsatzumgebung abhängig. Die Szenenmerkmale entstehen deshalb in einem komplett unüberwachten Lemprozeß, der die Wahmehmung des Roboters an die Eigenschaften von Umwelt und Sensoren anpaßt, während die Ultraschallmerkmale (24 Meßwerte) fest vorgegeben sind und „nur" die Aufteilung des Raums anhand dieser Merkmale selbstorganisierend erfolgt.

Obwohl hier keine Ergebnisse in dieser Richtung gezeigt werden, lassen sich jedoch auch beiden Sensortypen zur Situationsdifferenzierung kombinieren, um die jeweiligen Vorteile der verschiedenen Sensoren gleichzeitig zu nutzen.

\section{Zusammenfassung}

Der vorliegende Beitrag behandelt Aspekte des Lemens im Zusammenhang mit dem Aufbau von Umweltkarten durch mobile Serviceroboter. Eine lernende Steuerung ist dann erforderlich, wenn relevante Aspekte der Einsatzumgebung des Roboters zum Entwurfszeitpunkt unbekannt sind. Dies gilt gerade im Servicebereich sowohl für die topologische Struktur der Umwelt als auch für ihre sensorisch erfaßbaren Charakteristika. Deshalb brauchen lemende Serviceroboter auch eine lernende Sensordaten(vor)verarbeitung.

Nach einleitenden, prinzipiellen Überlegungen darüber, was eine intelligente und vor allem lernende
Robotersteuerung leisten muß, wurde zunächst für einen Roboter mit Ultraschallsensoren gezeigt, wie ein diesen Anforderungen entsprechendes System realisiert werden kann. Es wurde diskutiert, da $B$ für eine gegebene Sensorik ein Kompromiß zwischen Wiedererkennbarkeit und Erkennungsvielfalt in Bezug auf die Situationen, in denen sich das System befindet, gefunden werden muß. Von der Wahl dieses Kompromisses hängen die Stabilität des Kartenaufbauprozesses und die „Größe“ der kartierbaren Umwelt ab. Verbesserungen in dieser Beziehung lassen sich nur erreichen, wenn man leistungsfähigere Sensoren verwendet. Deshalb wurde im Anschluß demonstriert, wie bildbasierte Ortsrepräsentationen erlernt werden können. Dies wurde im Vergleich mit der Ultraschallösung diskutiert.

\section{Literatur}

[1] Burschka, D., Eberst, C., Hauck, A. und Stöffler, N. O. Hierarchische Umgebungsmodellierung für Lokalisation, Exploration und Objektidentifikation. In: Dillmann, R. und Lüth, T. (Hrsg.), Autonome Mobile Systeme (AMS'95), Informatik Aktuell, Seiten 132-141. Springer Verlag, Heidelberg, Dezember 1995.

[2] Kim, D. und Nevatia, R.: A Method for Recognition and Localization of Generic Objects for Indoor Navigation. In 1994 ARPA Image Understanding Workshop, Seiten 13-16, Monterey (USA), November 1994.

[3] Weckesser, P., Dillman, R., Elbs, M. und Hampel, S.: Multiple Sensor Processing for High-Precision Navigation and Environmental Modelling with a Mobile Robot. In Proceedings of the IEEE/RSJ International Conference on Intelligent Robots and Systems, Band 1, Seiten 453-458, Pittsburgh (USA), 1995

[4] Fäustle, P., Daxwanger, W. und Schmidt, G.: Steuerung lokaler Fahrmanöver durch direkte Kopplung abbildender Sensorik an ein künstliches neuronales Netz. In: Levi, P. und Bräunl, T. (Hrsg.), Autonome Mobile Systeme (AMS'94), Informatik aktuell, Seiten 214-225, Stuttgart, 1994. Springer Verlag, Heidelberg.

[5] Moravec, H. P. und Elfes, A.: High Resolution Maps from Wide Angle Sonar. In: International Conference on Robotics and Automation, Seiten 19-24, 1985.

[6] Kurz, A.: Lemende Steuerung eines autonomen mobilen Roboters - Untersuchungen zum Erwerb sensomotorischer Fähigkeiten und einer für Navigationszwecke nutzbaren Umweltkarte. Dissertation, Technische Hochschule Darmstadt, Düsseldorf, 1994.

[7] Schürmann, J.: Neuronale Netze und die klassischen Methoden der Mustererkennung. FhG-Berichte 1-1991, 1991.

[8] Kohonen, T.: Self-Organization and Associative Memory. Springer, New York, London, Paris, Tokyo, 1988.

[9] $v$. Wichert, G.: Selforganizing Visual Perception for Mobile Robot Navigation. In 1st Euromicro Workshop on Advanced Mobile Robots (EUROBOT'96), Seiten 194-200, Kaiserslautern, Germany, 1996. IEEE Computer Society Press.

[10] $v$. Wichert, G. und Kleiner, K.: Selbstorganisierende Bildanalyse für die Navigation von mobilen Robotem. In: Dillmann, R. und Lüth, T. (Hrsg.), Autonome Mobile Systeme (AMS'95), Informatik Aktuell. Springer Verlag, Heidelberg, Dezember 1995.

[11] $H u, M .-K$.: Visual Pattern Recognition by Moment Invariants. IRE Transactions on Information Theory, 8:179-187, 1961.

[12] Fritzke, B.: A Growing Neural Gas Network Learns Topologies. Andvances in Neural Information Processing Systems, 7, 1995.

Manuskripteingang: 16. Januar 1997.

Verfügbar unter

lediglich die vom Gesetz vorgesehenen Nutzungsrechte gemäß UrhG 